\title{
On some damped 2 body problems
}

\author{
Alain Haraux \\ Sorbonne Université, Université Paris-Diderot SPC, CNRS, INRIA, \\ Laboratoire Jacques-Louis Lions, LJLL, F-75005, Paris, France. \\ e-mail: haraux@ann.jussieu.fr
}

December 23, 2020 


\begin{abstract}
The usual equation for both motions of a single planet around the sun and electrons in the deterministic Rutherford-Bohr atomic model is conservative with a singular potential at the origin. When a dissipation is added, new phenomena appear. It is shown that whenever the momentum is not zero, the moving particle does not reach the center in finite time and its displacement does not blow-up either, even in the classical context where arbitrarily large velocities are allowed. Moreover we prove that all bounded solutions tend to 0 for $t$ large, and some formal calculations suggest the existence of special orbits with an asymptotically spiraling exponentially fast convergence to the center. A related model with exponentially damped central charge or mass gives some explicit exponentially decaying solutions which might help future investigations. An atomic contraction hypothesis related to the asymptotic dying off of solutions proven for the dissipative model might give a solution to some intriguing phenomena observed in paleontology, familiar electrical devices and high scale cosmology.
\end{abstract}

Key words: gravitation, singular potential, global solutions, spiraling orbit. 


\section{Introduction}

The usual equation for both motions of a single planet around the sun and electrons in the deterministic Rutherford-Bohr atomic model is conservative with a singular potential at the origin. When a dissipation is added to the basic equation

$$
m u^{\prime \prime}=-\frac{q^{2}}{4 \pi \varepsilon_{0}} \frac{u}{|u|^{3}}
$$

modelling Coulomb's central force (with $q$ the elementary charge, $\mathrm{m}$ the mass of the electron and $\varepsilon_{0}$ the vacuum permittivity) or its equivalent Newton's law for planets (where $G$ is the gravitational constant and $M_{S}$ the mass of the sun)

$$
u^{\prime \prime}=-G M_{S} \frac{u}{|u|^{3}}
$$

written in complex form in the orbital plane with a suitable set of axes and length unit, the global behavior of solutions will be changed and we expect that at least some solutions $u(t)$ will tend to 0 for $t$ large, giving a possible explanation for several evolutionary phenomena which remained misunderstood until now in several areas of Science. This leads us to consider, as the simplest possible dissipative perturbation of the conservative problem

$$
u^{\prime \prime}+c_{0} \frac{u}{|u|^{3}}=0
$$

including both equations, the modified ODE

$$
u^{\prime \prime}+\delta u^{\prime}+c_{0} \frac{u}{|u|^{3}}=0 .
$$

As we shall see, that equation does not have any solution for which $|u(t)|$ is an exact decreasing exponential $C e^{-\eta t}$. While looking for such solutions it is natural to investigate a slightly more complicated case where we allow the charge $q$ or the driving mass $M_{S}$ to decay exponentially in time. This leads to the class of equations

$$
u^{\prime \prime}+\delta u^{\prime}+c_{0} e^{-\alpha t} \frac{u}{|u|^{3}}=0
$$

for which explicit exponentially decaying solutions will exist in some particular cases.

In the present paper, we derive some partial results on (1.2) and (1.3) together with some heuristic discussion and potential application to the explanation of various natural phenomena. In section 2 we recall, to make subsequent comparisons easier, the Rutherford-Bohr atomic model with its circular orbits. in Section 3, we prove that whenever the momentum is not zero, the moving particle subject to equation (3.1) does not reach the center in finite time and its displacement does not blow-up in finite time either, even in the classical context where arbitrarily large velocities are allowed. In addition, we prove that $u(t)$ remains bounded under a smallness condition involving both initial radius and velocity. Finally it turns out that all bounded non-vanishing solutions converge to 0 at infinity in $t$. In Section 4, we prove that (1.2) does not have any solution for which $|u(t)|$ is an exact decreasing exponential $C e^{-\eta t}$. However some formal calculations suggest the existence of special orbits with an asymptotically spiraling 
convergence to the center. We conclude that section by a few heuristic remarks. In Section 5 , we show that equation (1.3) does have solutions for which $|u(t)|$ is an exact decreasing exponential $C e^{-\eta t}$ if, and only if $\alpha=\delta$ or $\alpha=\frac{3 \delta}{2}$. We give the corresponding explicit formulas. We conclude the paper, in Section 6, by explaining the motivation of this research which provides a very simplified model to explain a few still unexplained phenomena from paleontology, technology and cosmology: the atomic contraction hypothesis.

\section{Recalling the classical Rutherford-Bohr model.}

The Rutherford model (cf.[12]), which served as a basis for Bohr's model [1] of the hydrogen atom, is based on a corpuscular conception of protons and electrons and the application of Coulomb's law for electrostatic forces. Denoting by $q$ the common absolute value of the charges of proton and electron, the equation of motion, considering the unique proton of the nucleus as the center of coordinates, can be written in the plane of the orbit in complex form. Denoting by $u$ the position of the electron in the complex plane, we have

$$
m u^{\prime \prime}=-\frac{q^{2}}{4 \pi \varepsilon_{0}} \frac{u}{|u|^{3}} .
$$

The electron is travelling on a circular orbit

$$
|u(t)|=R
$$

so that the solutions take the form

$$
u(t)=R \exp \left(i \omega\left(t+t_{0}\right)\right.
$$

with

$$
\omega^{2}=\frac{q^{2}}{4 \pi \varepsilon_{0} m R^{3}}
$$

In particular the constant velocity of the electron is

$$
\left|u^{\prime}(t)\right|=v=K R^{-1 / 2}
$$

for some positive constant $K$. This is consistent with the analogous property given by the third Kepler's law for the planets motion driven by the gravitational field of the sun. In the framework of Bohr's modelization, it is assumed that the radius $R$ can take only the values of a sequence of the form $r_{n}=r_{0} n^{2}$ with $n$ a positive integer. In particular the electron cannot "fall" on the nucleus.

Bohr's theory explains neither why all electrons so luckily always choose a circular orbit rather than other elliptic possibilities, nor even how the first atoms of matter could appear, even in the simplest case of the hydrogen atom. If an electron is left without initial velocity in the close surrounding of a proton, an atom will never appear since the electron will collide with the proton in a very short time. The problem of the origin of the initial kinetic energy of the electron is left open. Besides, there is no explanation of why some circular orbits would be stable and not the others. An attempt to circumvent that problem is quantum mechanics leading to Schrödinger's equation (cf. [13]). For the time being we shall avoid quantum considerations, even though such a framework is presently considered necessary to understand the electromagnetic waves produced by hot matter (and therefore to consider spectral properties and the redshift problematics). 


\section{General results for equation (1.2)}

In this section, we investigate the standard relevant questions for the solutions of (1.2): wellposedness, boundedness and asymptotics.

\subsection{A global existence result}

For convenience we recall our basic equation

$$
u^{\prime \prime}+\delta u^{\prime}+c \frac{u}{|u|^{3}}=0
$$

where we simply wrote $c$ instead of $c_{0}$, since for mathematicians $c$ is not always the velocity of light. In order to solve this equation with $u=u(t) \in \mathbb{R}^{2}=\mathbb{C}$ we introduce the amplitude and the phase

$$
u(t)=r(t) e^{i \theta(t)}
$$

and we shall drop the variable $t$ when it does not lead to confusion. Here all derivatives are with respect to $t$. From the formulas

$$
\begin{gathered}
u^{\prime}=r^{\prime} e^{i \theta}+i r \theta^{\prime} e^{i \theta}=\left(r^{\prime}+i r \theta^{\prime}\right) e^{i \theta}=\left(r^{\prime}+i r \theta^{\prime}\right) \frac{u}{|u|}, \\
u^{\prime \prime}=\left[\left(r^{\prime \prime}-r \theta^{\prime 2}\right)+i\left(2 r^{\prime} \theta^{\prime}+r \theta^{\prime \prime}\right)\right] \frac{u}{|u|}
\end{gathered}
$$

we conclude that (3.1) is equivalent to the system of two real equations

$$
\begin{gathered}
r^{\prime \prime}-r \theta^{2}+\delta r^{\prime}+\frac{c}{r^{2}}=0 . \\
2 r^{\prime} \theta^{\prime}+r \theta^{\prime \prime}+\delta r \theta^{\prime}=0 .
\end{gathered}
$$

We observe first that when trying to solve (3.1) for $t \geq 0$, the initial value $u(0)=0$ is excluded by the singularity, while whenever $u(0) \neq 0$ we shall obtain at least a (unique as usual) local solution for any initial velocity $u^{\prime}(0)$. Moreover, multiplying (3.3) by $r$ we reduce it to

$$
\left(r^{2} \theta^{\prime}\right)^{\prime}+\delta r^{2} \theta^{\prime}=0
$$

so that

$$
\forall t \geq 0, \quad r^{2}(t) \theta^{\prime}(t)=M e^{-\delta t}
$$

with

$$
M=: r^{2}(0) \theta^{\prime}(0) \text {. }
$$

This relation expresses the variation of the momentum of the solution, which was constant in the conservative case $\delta=0$ and decays exponentially when $\delta>0$. By plugging in (3.2) the value of $\theta^{\prime}$ given by (3.4), we are left with an equation involving $r(t)$ only:

$$
r^{\prime \prime}-\frac{M^{2} e^{-2 \delta t}}{r^{3}}+\frac{c}{r^{2}}+\delta r^{\prime}=0
$$

We now state our first result 
Theorem 3.1. Assuming $M \neq 0$, the unique local non-vanishing solution $r=r(t)$ of (3.5) with any initial data $r(0)=r_{0} \neq 0 ; r^{\prime}(0)=r_{0}^{\prime} \in \mathbb{R}$ is global and we have for some constants $C>0, \eta>0$

$$
\begin{array}{ll}
\forall t \geq 0, & \left|r^{\prime}(t)\right| \leq C e^{\delta t} \\
\forall t \geq 0, & r(t) \geq \eta e^{-2 \delta t} .
\end{array}
$$

Proof. The existence and uniqueness of local non-vanishing solutions is obvious. Let us introduce the scalar function $F$, defined as long as the solution $r$ exists and does not vanish by the formula:

$$
F(t):=\frac{r^{\prime 2}(t)}{2}-\frac{c}{r(t)}+\frac{M^{2}}{2} \frac{e^{-2 \delta t}}{r^{2}(t)} .
$$

We have immediately

$$
F^{\prime}(t)=r^{\prime}(t)\left(r^{\prime \prime}(t)+\frac{c}{r^{2}(t)}-\frac{M^{2} e^{-2 \delta t}}{r^{3}(t)}\right)-\frac{M^{2} \delta e^{-2 \delta t}}{r^{2}(t)}=-\delta r^{\prime 2}(t)-\frac{M^{2} \delta e^{-2 \delta t}}{r^{2}(t)}<0 .
$$

In particular for all $t \in[0, \operatorname{Tmax})$ we have

$$
\frac{r^{\prime 2}(t)}{2}-\frac{c}{r(t)}+\frac{M^{2}}{2} \frac{e^{-2 \delta t}}{r^{2}(t)} \leq F_{0}:=F(0)
$$

which can be rearranged in the more convenient form

$$
\frac{r^{\prime 2}(t)}{2}+\frac{M^{2}}{2}\left(\frac{e^{-\delta t}}{r(t)}-\frac{c}{M^{2}} e^{\delta t}\right)^{2} \leq F_{0}+\frac{c^{2}}{2 M^{2}} e^{2 \delta t}
$$

providing at once the two inequalities

$$
\forall t \in[0, \text { Tmax }), \quad r^{\prime 2}(t) \leq 2\left(F_{0}+\frac{c^{2}}{2 M^{2}} e^{2 \delta t}\right) \leq\left(2 F_{0}+\frac{c^{2}}{M^{2}}\right) e^{2 \delta t}
$$

and

$$
\forall t \in[0, \operatorname{Tmax}), \quad \frac{e^{-\delta t}}{r(t)} \leq \frac{c}{M^{2}} e^{\delta t}+\sqrt{\frac{2}{M^{2}} F_{0}+\frac{c^{2}}{M^{4}} e^{2 \delta t}} \leq K e^{\delta t} .
$$

The conclusions follow immediately with

$$
C=\left(2 F_{0}+\frac{c^{2}}{M^{2}}\right)^{1 / 2} ; \quad \eta=\frac{1}{K} .
$$

Corollary 3.2. Assume

$$
\operatorname{Im}\left(\overline{u_{0}} u_{0}^{\prime}\right) \neq 0
$$

Then the local solution of (3.1) with initial conditions $u(0)=u_{0} ; u^{\prime}(0)=u_{0}^{\prime}$ is global and satisfies for some constants $D>0, \eta>0$

$$
\begin{aligned}
& \forall t \geq 0, \quad\left|u^{\prime}(t)\right| \leq D e^{\delta t} . \\
& \forall t \geq 0, \quad|u(t)| \geq \eta e^{-2 \delta t} .
\end{aligned}
$$


Proof. I suffices to prove that the two above inequalities are satisfied for all $t \in[0$, Tmax $)$, the maximal existence time for a non-vanishing solution $u$ of (3.1) with initial conditions $u(0)=u_{0} ; u^{\prime}(0)=u_{0}^{\prime}$. Selecting for $\theta$ a continuous determination of the argument of $u(t)$, the pair $(r, \theta)$ satisfies the equivalent system of equations (3.2)-(3.3). In addition an immediate calculation shows that $\theta^{\prime}=\operatorname{Im}\left(\frac{\bar{u}}{|u|} u^{\prime}\right)$ and in particular

$$
\operatorname{Im}\left(\overline{u_{0}} u_{0}^{\prime}\right)=r(0) \theta^{\prime}(0)
$$

By the previous theorem, the solution $r(t)$ of the scalar equation for the radius is global and satisfies the concluding inequalities. Then the pair $(r, \theta)$ correspond to the non-vanishing solution $u$ of (3.1) with initial conditions $u(0)=u_{0} ; u^{\prime}(0)=u_{0}^{\prime}$ which is therefore global with $|u(t)| \geq \eta e^{-2 \delta t}$. For the last inequality we observe that

$$
\mid \theta^{\prime}(t) r(t) \leq M e^{-\delta t} r^{-1}(t) \leq \frac{M}{\eta} e^{\delta t}
$$

Then

$$
\left|u^{\prime}(t)\right|=\left|r^{\prime}+i r \theta^{\prime}\right| \leq\left|r^{\prime}(t)\right|+\left|r(t) \theta^{\prime}(t)\right| \leq\left(C+\frac{M}{\eta}\right) e^{\delta t}:=D e^{\delta t}
$$

\subsection{Bounded trajectories}

The next result does not require that $M \neq 0$.

Theorem 3.3. Let $u_{0} \neq 0$ and assume the initial smallness condition

$$
\left|u_{0}\right|\left|u_{0}^{\prime}\right|^{2}<2 c .
$$

Then the local solution $u$ of $(3.1)$ on $[0, T)$ with initial conditions $u(0)=u_{0} ; u^{\prime}(0)=u_{0}^{\prime}$ satisfies the inequality

$$
\forall t \in[0, T), \quad|u(t)| \leq \frac{2 c\left|u_{0}\right|}{2 c-\left|u_{0}\right|\left|u_{0}^{\prime}\right|^{2}} .
$$

In particular if $u$ does not vanish in finite time, $u$ is a global bounded non-vanishing solution.

Proof. We start from the inequality

$$
\frac{r^{\prime 2}(t)}{2}-\frac{c}{r(t)}+\frac{M^{2}}{2} \frac{e^{-2 \delta t}}{r^{2}(t)} \leq F_{0}=\frac{r_{0}^{\prime 2}}{2}-\frac{c}{r_{0}}+\frac{M^{2}}{2 r_{0}^{2}}
$$

and we observe that if $F_{0}<0$, the inequality $\frac{c}{r(t)} \geq-F_{0}=\left|F_{0}\right| \operatorname{implies} r(t) \leq \frac{c}{\left|F_{0}\right|}$. Now we compute $F_{0}$ in terms of the initial data. In fact we have the formula

$$
\left|u^{\prime}\right|^{2}=r^{\prime 2}+\theta^{\prime 2} r^{2}=r^{\prime 2}+\frac{M^{2}}{r^{2}}
$$

so that for $t=0$ we find

$$
F_{0}=\frac{\left|u_{0}^{\prime}\right|^{2}}{2}-\frac{c}{r_{0}}=\frac{\left|u_{0}^{\prime}\right|^{2}}{2}-\frac{c}{\left|u_{0}\right|}
$$


therefore the condition $F_{0}<0$ is equivalent to $\left|u_{0}\right|\left|u_{0}^{\prime}\right|^{2}<2 c$. The previous observation now gives that under condition (3.11), we have

$$
r(t) \leq \frac{c}{\frac{c}{\left|u_{0}\right|}-\frac{\left|u_{0}^{\prime}\right|^{2}}{2}}=\frac{2 c\left|u_{0}\right|}{2 c-\left|u_{0}\right|\left|u_{0}^{\prime}\right|^{2}} .
$$

\subsection{Convergence to 0 of non-vanishing bounded solutions}

As in the case of the conservative equation (1.1) which is well known to have hyperbolic and parabolic trajectories, we suspect that (3.1) may have some unbounded solutions. The next result shows, on the other hand, that in sharp constrast with (1.1), (3.1) does not have any periodic trajectory at all.

Theorem 3.4. For any solution $u$ of (3.1) such as $|u(t)|$ is global, positive and bounded on $\mathbb{R}^{+}$, we have

$$
\lim _{t \rightarrow+\infty}|u(t)|=0
$$

Proof. We introduce the total energy

$$
E(t):=\frac{1}{2}\left|u^{\prime}\right|^{2}(t)-\frac{c}{|u(t)|} .
$$

Since $u$ never vanishes, it is clear that $u \in C^{2}\left(\mathbb{R}^{+}\right)$and we have

$$
E^{\prime}(t)=-\delta\left|u^{\prime}(t)\right|^{2}
$$

In particular, $E(t)$ is non-increasing. Then we have two possibilities

\section{Case 1}

$$
\lim _{t \rightarrow+\infty} E(t)=-\infty
$$

Then since $\frac{c}{|u(t)|} \geq-E(t)$ we conclude that

$$
\lim _{t \rightarrow+\infty}|u(t)|=0
$$

\section{Case 2}

$$
\lim _{t \rightarrow+\infty} E(t)=E^{*}>-\infty
$$

Then $E(t)$ is bounded and

$$
\forall t \in \mathbb{R}^{+}, \quad E(0)-E(t)=\delta \int_{0}^{t} \mid u^{\prime}\left(\left.s\right|^{2} d s .\right.
$$

In particular $u^{\prime} \in L^{2}\left(\mathbb{R}^{+}, \mathbb{C}\right)$. We have assumed that $u(t)$ is bounded, hence precompact in $\mathbb{R}^{+}$ with values in $\mathbb{C}$. Therefore if (3.13) is not satisfied we may assume that for some sequence $t_{n}$ tending to $+\infty$

$$
\lim _{n \rightarrow+\infty} u\left(t_{n}\right)=w \neq 0
$$


On the other hand we have

$$
\lim _{n \rightarrow+\infty} u^{\prime}\left(t_{n}+s\right)=0
$$

in the strong topology of $L^{2}(0,1)$ and in particular, by Cauchy-Schwarz-inequality we find

$$
\lim _{n \rightarrow+\infty} u\left(t_{n}+s\right)=w
$$

uniformly on $[0,1]$. Since $w \neq 0$ this implies

$$
\lim _{n \rightarrow+\infty} c \frac{u\left(t_{n}+s\right)}{\left|u\left(t_{n}+s\right)\right|^{2}}=c \frac{w}{|w|^{3}}
$$

uniformly on $[0,1]$. But then by the equation

$$
\lim _{n \rightarrow+\infty} u^{\prime \prime}\left(t_{n}+s\right)=c \frac{w}{|w|^{3}}
$$

in the strong topology of $L^{2}(0,1)$. This is contradictory with (3.18). Indeed it implies for instance

$$
\lim _{n \rightarrow+\infty} \int_{0}^{1} s(1-s) u^{\prime \prime}\left(t_{n}+s\right) d s=c \int_{0}^{1} s(1-s) d s \frac{w}{|w|^{2}}=z \neq 0
$$

while on the other hand

$$
\int_{0}^{1} s(1-s) u^{\prime \prime}\left(t_{n}+s\right) d s=-\int_{0}^{1}(1-2 s) u^{\prime}\left(t_{n}+s\right) d s \longrightarrow 0 .
$$

This contradiction concludes the proof.

As an immediate consequence of the previous theorem and the results of Sections 2 and 3, we obtain

Corollary 3.5. Assume $\operatorname{Im}\left(\overline{u_{0}} u_{0}^{\prime}\right) \neq 0$ and $\left|u_{0} \| u_{0}^{\prime}\right|^{2}<2 c$. Then the local solution of (3.1) with initial conditions $u(0)=u_{0} ; u^{\prime}(0)=u_{0}^{\prime}$ tends to 0 as $t$ tends to infinity.

\section{About exponentially decaying solutions for (1.2)}

The previous theorem, proved by contradiction, is not constructive and therefore does not provide any hint on the rate of decay to 0 of bounded solutions. The question naturally arises of the existence of exponentially decaying solutions. First of all can we have $|u(t)|=C e^{-\eta t}$ ? Plugging $r(t)=C e^{-\eta t}$ in (3.5) we find

$$
\left(\eta^{2}-\delta \eta\right) C+\frac{c}{C^{2}} e^{3 \eta t}-\frac{M^{2}}{C^{3}} e^{-2 \delta+4 \eta t} \equiv 0
$$

which implies both $\eta^{2}=\eta \delta$ and $\eta=2 \delta$. This is impossible. 


\subsection{A formal calculation for spiraling solutions}

We now try a formal calculation suggesting that solutions with norm equivalent to a decreasing exponential for $t$ large might however exist. We would like to see which sort of solutions would replace the circular trajectories of the conservative problem (1.1) (cf. also section 2). Observing that such trajectories correspond to a constant angular velocity and for them the nonlinear term of the radial equation vanishes, it is natural to try

$$
r(t)=\frac{M^{2}}{c} e^{-2 \delta t}
$$

By the argument above his cannot be an exact solution of the equation and indeed

$$
r^{\prime \prime}+\delta r^{\prime}=\frac{M^{2}}{c} 2 \delta^{2} e^{-2 \delta t} \neq 0
$$

but at least the result is non-singular and even becomes very small if $\delta$ is small. This gives the idea to look for a solution of the form

$$
r(t)=\frac{M^{2}}{c} e^{-2 \delta t}+\varepsilon(t)
$$

where $\varepsilon(t)$ would be negligible with respect to $\frac{M^{2}}{c} e^{-2 \delta t}$, either uniformly, or at least for $t$ large. Plugging this in the equation we find

$$
c \varepsilon(t)=-r^{3}\left(r^{\prime \prime}+\delta r^{\prime}\right)
$$

If now we replace both

$$
r^{3} \sim \frac{M^{6}}{c^{3}} e^{-6 \delta t} ; \quad r^{\prime \prime}+\delta r^{\prime} \sim \frac{M^{2}}{c}\left(2 \delta^{2}\right) e^{-2 t}
$$

by their alleged "main part" we find

$$
\varepsilon(t) \sim-2 \delta^{2} \frac{M^{8}}{c^{5}} e^{-8 \delta t}
$$

which is indeed quite negligible with respect to $\frac{M^{2}}{c} e^{-2 \delta t}$ for $t$ large and even uniformly if $\delta$ and $\frac{M^{6}}{c^{4}}$ are both small enough. To see the rule we main consider trying the next step. the calculations are slightly more involved but we get rather easily

$$
\varepsilon(t) \sim-2 \delta^{2} \frac{M^{8}}{c^{5}} e^{-8 \delta t}+124 \delta^{4} \frac{M^{14}}{c^{9}} e^{-14 \delta t} \ldots
$$

From the algebraic point of view, it is not difficult to see that the process can be continued indefinitely with the appearance of a single new exponential term at each step. So that we can dream of a solution of the form

$$
r(t)=\frac{M^{2}}{c} \sum_{n=0}^{\infty}(-1)^{n} C_{n} \delta^{2 n}\left(\frac{M^{6}}{c^{4}}\right)^{n} e^{-(2+6 n) \delta t}
$$

The only difficulty for convergence would be to control the growth of $C_{n}$. Actually $C_{n}$ seems to grow like a factorial, precluding any sort of convergence for any fixed value of $t$. Then in which sense would the above calculations approach a solution? At each step we have an approximate solution with a very close accuracy for $t$ large. For the time being the moral of the story remains a mystery for the author. Could it be that the above formula is the Taylor expansion of an actual (non-analytic) solution? In any case it is clear that a different method is needed to find the solutions we are looking for. 


\subsection{Some remarks}

The previous calculations suggest the possible existence of special orbits with an asymptotically (exponentially fast) spiraling convergence to the center. More precisely if we succeed in finding, by whichever method, a solution $r$ of (3.5) with

$$
r(t) \sim C e^{-2 \delta t}
$$

for a certain $C=C(M)>0$, from this we can build a solution $u=r e^{i \theta}$ with

$$
\theta^{\prime 2} \sim K e^{6 \delta t}
$$

so that $\left|u^{\prime}\right|^{2}=r^{\prime 2}+\theta^{\prime 2} r^{2} \geq k e^{2 \delta t}$ for some positive $k$ and $t$ large. The kinetic energy blows-up exponentially in such a case, which is not absurd since the potential energy also.

Remark 4.1. This construction seems interesting, but even in case it works this is not entirely satisfactory for the following reason: the family of spiraling orbits built in this way would depend on only two real parameters (the moment and the initial phase) whereas the phase space has four dimensions.

Remark 4.2. For the moment, despite the formal calculation of the previous section, we know nothing about the rate of decay of any solution to 0 . The only certain information that we have on asymptotics is the inequality $r(t) \geq \eta e^{-2 \delta t}$ for some $\eta>0$. This inequality can be refined asymptotically, relying on the inequality

$$
\forall t \in[0, T \max ), \quad \frac{e^{-\delta t}}{r(t)} \leq \frac{c}{M^{2}} e^{\delta t}+\sqrt{\frac{2}{M^{2}} F_{0}+\frac{c^{2}}{M^{4}} e^{2 \delta t}}
$$

to yield

$$
\varliminf_{t \rightarrow \infty} r(t) e^{2 \delta t} \geq \frac{M^{2}}{2 c}
$$

Remark 4.3. In the case $M=0$, it should be possible to determine whether, as in the conservative case, solutions with initial velocity directed towards the center vanish in finite time, if necessary under some additional conditions. We did not study this question here since it is not our main concern.

\section{$5 \quad$ Partial results on equation (1.3)}

We now turn to the case where the charge or the mass of the central object decays exponentially with $t$. We look for solutions of (1.3) of the form

$$
u(t)=U e^{-\varepsilon t} e^{i v(t)}
$$

Then

$$
u^{\prime}=\left(-\varepsilon+i v^{\prime}\right) u ; \quad u^{\prime \prime}=\left[i v^{\prime \prime}+\left(-\varepsilon+i v^{\prime}\right)^{2}\right] u ; \quad \frac{u}{|u|^{3}}=U^{-3} e^{3 \varepsilon t} u
$$

and (1.3) reduces to the system

$$
v^{\prime \prime}-2 \varepsilon v^{\prime}+\delta v^{\prime}=\varepsilon^{2}-v^{\prime 2}-\delta \varepsilon+U^{-3} c_{0} e^{(-\alpha+3 \varepsilon) t}=0 .
$$


The first equation shows an exact exponential variation of $v^{\prime}$ (which might be constant when the decrement is 0 ), and then for the second equation to be consistent we need that either $\varepsilon^{2}=\delta \varepsilon$ or $-\alpha+3 \varepsilon=0$. In the second case we derive $2 \varepsilon=\delta$.

Case 1. For $\varepsilon=\delta$, we find $v^{\prime}=v^{\prime}(0) e^{\delta t}$ and $v^{\prime 2}=U^{-3} c_{0} e^{(-\alpha+3 \delta) t}$, so that $\alpha=\delta$ and $v^{\prime 2}(0)=U^{-3} c_{0}$. This yields a family of anti-clockwise rotating solutions of the form

$$
u_{+}(t)=U e^{-\delta t} \exp \left(i \frac{\sqrt{c_{0}}}{\delta U^{3 / 2}} e^{\delta t}+i \phi\right)
$$

depending on the two arbitrary parameters $U$ and $\phi$ as in the case of circular orbits of (1.1). We also have clockwise rotating solutions

$$
u_{-}(t)=U e^{-\delta t} \exp \left(-i \frac{\sqrt{c_{0}}}{\delta U^{3 / 2}} e^{\delta t}+i \phi\right) .
$$

These are solution with exponentially fast phase, the kinetic energy of electrons is

$$
\frac{m}{2}\left|u^{\prime}(t)\right|^{2}=\frac{m}{2}\left(\varepsilon^{2}+v^{\prime 2}(t)\right)|u(t)|^{2}=\frac{m}{2}\left(c_{0} U^{-1}+\varepsilon^{2} U^{2} e^{-2 \varepsilon t}\right)
$$

which tends to the constant $\frac{m}{2} c_{0} U^{-1}$ for $t$ large. Of course the acceleration becomes infinite in norm.

Case 2. For $\varepsilon=\frac{\delta}{2}$, we find $\alpha=3 \varepsilon=\frac{3}{2} \delta, v^{\prime \prime}=0$ and $v^{\prime 2}=U^{-3} c_{0}+\frac{\delta^{2}}{4}$. This yields a family of anti-clockwise rotating solutions of the form

$$
u_{+}(t)=U e^{-\frac{\delta}{2} t} \exp \left(i t \sqrt{U^{-3} c_{0}+\frac{\delta^{2}}{4}}+i \phi\right)
$$

depending on the two arbitrary parameters $U$ and $\phi$ as in the case of (3.1). We also have clockwise rotating solutions

$$
u_{-}(t)=U e^{-\frac{\delta}{2} t} \exp \left(-i t \sqrt{U^{-3} c_{0}+\frac{\delta^{2}}{4}}+i \phi\right) .
$$

In that case the kinetic energy of electrons, given by

$$
\frac{m}{2}\left|u^{\prime}(t)\right|^{2}=\frac{m}{2}\left(\varepsilon^{2}+v^{\prime 2}(t)\right)|u(t)|^{2}=\frac{m}{2} e^{-\delta t}\left(c_{0} U^{-1}+\frac{\delta^{2}}{2} U^{2}\right)
$$

tends to 0 exponentially. Here also the acceleration becomes infinite in norm.

Remark 5.1. (Asymptotics for small values of the dissipation and aging constants.) It is easy to see on the formulas that with the specific choices of the aging constant $\alpha$ (equal to the damping constant or $3 / 2$ of its value), when $\delta$ tends to zero, we recover the classical circular orbits of (1.1) described in Section 2. The calculation is obvious in the case $\varepsilon=\frac{\delta}{2}$, and in the case $\varepsilon=\delta$ we use the convergence of $\frac{e^{\delta t}-1}{\delta}$ to $t$ as $\delta$ tends to 0 . In that case an infinite shift of the phase is necessary to recover the circular orbits.

Remark 5.2. It would certainly be interesting to investigate for the more complicated equation (1.3) the same basic questions (well-posedness and rough asymptotics) as for (1.2). But for the moment we refrained from doing this since the study of the smpler problem (1.2) is not yet complete. 
Remark 5.3. After the simple model of Rutherford-Bohr introduced in [1, 12] and after the introduction of undulatory mechanics by L. De Broglie, the purely probabilistic model of E. Schrödinger (cf. [13]) has been considered the best atomic model since 1926. It does not seem obvious at all to introduce a damping mechanism in that model, and it might very well happen that in order to do that, an effective coupling between deterministic corpuscular and probabilistic wave conceptions of particles has to be devised, as was always advocated by L. De Broglie and even suggested by E. Schrödinger himself in [13].

\section{The atomic contraction hypothesis.}

Here we are leaving mathematics for "natural philosophy". The calculations performed in this short mathematical report were in fact motivated by three concrete phenomena which until now have received rather unsatisfactory explanations, more precisely: some disturbing long term deformations of electrical appliances, the appearant gigantism of arthropods during the Carboniferous period, and the redshift of distant galaxies. The sudden birth of the (mightbe unfounded and somewhat arbitrary) atomic shrinking hypothesis is explained in a more detailed fashion in [7] from which we borrowed the calculations concerning equation (1.3). We recall here the context of [7].

\subsection{A disturbing concrete observation.}

Very recently, the author found in a drawer a small electrical device which had been forgotten there for about 10 years. It was obviously time to change the batteries, but when he took off the old ones, they came out much too easily. Actually there was no contact left between the batteries and the rigid metallic parts which were supposed to drive the current to the appliance. Did the batteries shrink or did the plastic compartment increase in size? Well, let us see with new batteries to decide: the problem turned out to be exactly the same. The compartments of both batteries seem to have been enlarged by something like 0.3 millimeters, and to re-establish the contact he interposed some small pieces of aluminum paper. It would be enough until he finds a more elegant solution. But what happened during that interval of 10 years? There has been no water in the drawer, no overheating, the electrical circuit of the appliance was left open since to action it you must press a button.

All of a sudden, he remembered that this kind of problems already happened to him several times, and the circumstances were always the same: an electrical device forgotten for 1 or 2 decades, the batteries were cylindrical, the compartments made of plastic, the contacts made of semi-rigid metallic blades. What is going on there?

\subsection{A fancy counter-model for the expansion of universe.}

In a flash the author made the connection with a somewhat absurd idea which stroke him a few weeks before: what if the expansion of universe were an illusion, and the reality would be contraction of matter? One may argue that in such a case there would be no redshift. Is it so clear? So 2 questions are in order : how to recover the redshift in the case of matter contraction, and what would be the mechanism involved in contraction? A first possibility to investigate is that contraction of matter is at the atomic level, a reduction of the distance between electrons and nucleons compared with the size of a proton. In such a case, metals 
should shrink at exactly the same speed as atoms, while materials involving some empty space between atoms could shrink more slowly, for instance polymerized plastic materials. This would explain the problem with batteries.

\subsection{The connection with carboniferous gigantism.}

During the carboniferous era, according to what was found by the specialists, gigantic arthropods such as giant dragonflies, roaches and centipedies (the arthropleura for instance) and many others coexisted with insects of normal size which have been found perfectly conserved inside amber. It is rather difficult to understand how some species of insects could have a size comparable to their present analogs, while some others were larger by almost one order of magnitude, which implies that their volume (and maybe weight, but maybe not according to the subsequent analysis...) should be several hundreds of times larger. This somehow contradicts the fact that species usually stabilize around a certain average size taking account of the fact that similarity laws for the numerous parameters deciding the size are usually different and natural selection leads to convergence of the size. The first remarks in the direction of size optimization go back to Galileo Galilei in his famous book "Two New Sciences " ([5]) comparing the shapes of the legs of elephants and smaller mammals such as dogs. To take another striking example, if you multiply the three dimensions of a gerris by 10 and keep its density equal to the present one, it will no longer be able to walk on the surface of water.

The currently admitted explanation for arthropod gigantism is the high proportion of oxygen in the air during the carboniferous era (cf. e.g. [8, 10]), which would have given a selective advantage and a capacity to grow much larger in particular to those arthropods with aquatic larvas, but this does not seem to apply to roaches and does not explain anything about giant plants. It is important to observe here that (to the author's knowledge) giants arthropods as well as giant plants did not arrive to us as solid massive objects but that we only found their fossilized contours.

It might be that in fact the insects and the plants of the carboniferous period had a normal size with respect to their times environment (all atoms being at that time huge compared to now) and, since their skeleton was made of polymerized chitin (respectively cellulose in the case of plants), they would have shrunk more slowly than stone and metal, exactly like the plastic compartments of electric appliances. In this case we clearly end up with huge fossile contours and the paradox disappears.

The "giant insects" and also "giant plants" were identified by their traces in sediments which accumulated in some regions which were before covered with water. So most probably the insects and plants were immersed after death and this gave to their polymerized part the time to inflate with comparison to metals or even liquids were atoms are very close to each other. Actually they did not inflate but they contracted more slowly. The experience with the batteries recalled in the first section suggests a logarithmic decrement for the size of atoms of about $10^{-3}$ when the time unit is the year. Present estimates on the time of recycling of plastic materials turn around 1000 years, but when the substance is confined, the phenomenon slows down since the bacteria need some time to arrive on the place. In 1000 years the maximum expansion would be a multiplication by e, and in 2000 years by $e^{2} \sim 8$. After the sediments surround the fossile constrained by its inflated size (compared to hard objects), the fossile 
itself stops growing and essentially disappears, since after that millions of years elapsed. We are left with a fossilized silhouette which will look between 5 and ten times its real size when we discover it.

\subsection{Main weak points of the model}

It is a bit too much to speak of a "model" when so many basic questions are still unsolved. But there are even worse points that it would be dishonest to hide here.

1) In a context where the size of atoms is suspected to vary with time, the question of unit length itself becomes problematic. Choosing the size of the proton as the reference is delicate, since it means that we consider it constant. In particular in the model (1.3) where the unit charge is subject to decay, one might wonder if the size of protons is such a good choice...

2) If our atoms shrink by a factor 2 every 1000 years, our world should disappear very soon. Because in 20000 years the electrons would reach the nuclei! Therefore we must imagine that a kind of barrier prevents this as it seems to be the case for gravitational forces.

3) The redshift of distant luminous matter (cf. $[9,14])$ has to be explained. In the past the atoms were larger with an exponential growth when we go backwards in time. We might imagine that the wavelength of electromagnetic radiations increase with the size of atoms, but Hubble's law then requires that the wavelength should be a logarithmic function of the radius of atoms. We have no idea of how such a phenomenon might take place. However we must here mention that some physicists (cf.e.g.[11]) consider an exponential expansion of space, in which case the appearent space expansion would fit with an exponential shrinking of atoms.

4) Last but not the least. Depending on when the shrinking phenomenon started, an exponential growth of atoms in the distant past leads to absurd conclusions. Although the size of atoms does not affect the total mass of planets and stars, beyond a certain size we end up with interpenetration of the planets and the sun, and much before that the dynamics of the solar system becomes difficult to understand...

Remark 6.1. It may now happen that the atomic contraction is more subtle than a simple exponential decay. A more thorough study of the models (1.2) and (1.3) for a small with respect to $\delta$ might reveal a more complex dynamics than a constant contraction factor by time unit. In that case we cannot really assume an exponential growth of atoms when the direction of time is reversed.

\subsection{The possible causes of atomic shrinking}

In the purely corpuscular, deterministic modelization of atoms, it is not too difficult to conceive: a very weak linear dissipation might typically result from random shocks of the rotating electrons with wandering particles, and it is not even necessary to change our vision of vacuum to admit the existence of such a damping: isolated particles such as neutrinos, photons or the electrons of wandering isolated hydrogen atoms might intereract with electrons and produce the same damping effect as a gas at a very large time scale.

It is also possible to imagine that the "gas" producing the damping is of a still unknown nature, for instance in the framework of a revised Fatio de Duillier-Lesage theory of gravitation (cf $[2,3,4]$ ) where gravitation is the result of the pressure of a presently unidentified gas of immaterial corpuscules. In [6], the author made the hypothesis that the pushing effect leading 
to gravity concerns the nucleons only. But it might happen that another kind of interaction (or even the same) with electrons finally lead to atomic contraction for very large times.

Let us finally observe that the pure deterministic Rutherford-Bohr model is known to be too simple and in particular has no chance to allow establishing a connection between the alledged atomic shrinking and the redshift of distant luminous matter. After a more thorough study of (1.2), assuming that the results are in some rough concordance with observations, the most difficult step will have to be done, more precisely a partially deterministic variant of Schrödinger's model will have to be devised and serious problems will only start at this level.

\section{Conclusion.}

It is always somewhat difficult to question a theory which has required many years of research of our best specialists worldwide. From the conceptual point of view the difficulties are considerable (for instance the specialists did not reintroduce until now the corpuscle aspect of elementary particles in the equations, and distinguishing between ground states and excited states in a time variable context is a quite challenging question) even if at some point we might become certain that the phenomenon is real. If it is the case, there will be many potential applications to technology since we could start understanding quite a few breakdowns which occur here and there when we use sophisticated machines during many years. Even if the theory becomes clear, it will never be simple because the present basic theory of matter, although probably incomplete, is already quite involved. In such a situation, if there is some benefit to gain from the atomic contraction hypothesis, computers will play an essential role to improve our technology and increase the duration of sophisticated equipments associating different kinds of materials. 


\section{References}

[1] N.Bohr, On the Constitution of Atoms and Molecules, Philosophical Magazine, Series 26, vol. 1, (July 1913) , p.1-24.

[2] Н. Снавот; Georges-Louis Lesage (1724-1803): a theoretician of gravitation in search of legitimacy, Arch. Internat. Hist. Sci. 53 (2003), no. 150-151, 157-183.

[3] M.R. Edwards; Pushing gravity: New perspectives on Lesage's theory of gravitation Apeiron, 2002, In: Revue d'histoire des sciences, tome 58, n2, 2005. pp. 519-520.

[4] M..R. EDWARds; Photon-graviton recycling as cause of gravitation Apeiron.14, 3 (2007), $214-230$.

[5] G. Galilei ; Two new sciences, A new translation with introduction and notes by Stillman Drake, The University of Wisconsin Press (1974).

[6] A. Haraux, About Dark Matter and Gravitation. Preprints 2020, 2020070198 (doi: 10.20944/preprints202007.0198.v1).

[7] A. Haraux, On Carboniferous Gigantism and Atomic Shrinking. Preprints 2020, 2020110544 (doi: 10.20944/preprints 202011.0544.v2).

[8] J.F.Harrison, A.Kaiser and J.M. VandenBrooks; Atmospheric oxygen level and the evolution of insect body size. Proceedings of the Royal Society B. 277(2010):1937-1946. doi:10.1098/rspb.2010.0001

[9] E.Hubble, M.L. Humason, The Velocity-Distance Relation among Extra-Galactic Nebulae, Astrophysical Journal, vol. 74, p. 43.

[10] Ryssa Parks; An Overview of Hypotheses and Supporting Evidence Regarding Drivers of Insect Gigantism in the Permo-Carboniferous, Western Washington University reports, Spring 2020.

[11] R. Penrose ; The Big Bang and its Dark-Matter Content: Whence, Whither, and Wherefore, Found Phys (2018) 48:1177-1190.

[12] E. Rutherford; The Scattering of $\alpha$ and $\beta$ Particles by Matter and the Structure of the Atom, E. Rutherford, F.R.S. Philosophical Magazine, Series 6, vol. 21 May 1911, p. 669-688

[13] E. Schrodinger; An Undulatory Theory of the Mechanics of Atoms and Molecules, Phys. Rev. vol. 28, no 6, decembre 1926, p. 1049-1070 (DOI 10.1103/PhysRev.28.1049)

[14] F. Zwicky; The redshift of extragalactic nebulae, Helvetica Physica Acta, Vol. 6 (1933), 110-127. 